\title{
On Cabannes' 32-Velocity Models of the Boltzmann Equation
}

\author{
By \\ Shuichi KAWASHIMA*, Akemi WATANABE*, \\ Machi MAEJI** and Yasushi SHIZUTA*
}

\section{§1. Introduction}

In the preceding papers [8], [9], we studied the general theory of the discrete Boltzmann equation and formulated several conditions under which the solutions in the large for the Cauchy problem exist and approach the Maxwellian corresponding to the initial data as $t \rightarrow \infty$. (One of these conditions which we shall use in this paper will be called simply the stability condition for the discrete Boltzmann equation.) Also we treated in [9] the 14-velocity model as an application of the results. We continue in this paper the study of concrete discrete models. Our aim is to verify the stability condition for the 32-velocity model introduced by Cabannes [4]. Since the size of the model is relatively large, the computation needed becomes necessarily lengthy.

First we recall the definition of the 14-velocity model. We consider a cube centered at the origin of the velocity space. The set of eight vertices of the cube defines an element model $M_{c}$. ( $A$ discrete model is said to be an element model when the moduli of the velocities are equal.) The centers of six surfaces of the cube form an octahedron and define another element model $M_{0}$. We introduce here the definition of the similarity. Two discrete models are said to be similar if one equals the other after the multiplication by a suitable positive constant. Let $\mathscr{F}_{c}$ and $\mathscr{F}_{o}$ be the families of discrete models similar to $M_{c}$ and $M_{o}$, respectively. Let us consider a

Communicated by S. Hitotumatu, November 30, 1985. Revised January 13, 1986.

*Department of Mathematics, Nara Women's University, Nara 630, Japan

**Division of Systems and Information Science, Central Research Laboratory, Mitsubishi Electric Corporation, Tsukaguchi, Amagasaki 661, Japan 
compound model of these two kinds of element models. We denote the moduli of the velocities of two element models by $R_{c}$ and $R_{o}$, respectively. In other words, $R_{c}$ and $R_{o}$ are the radii of the circumspheres of the cube and the octahedron corresponding to these models. Then the mixed collisions exist if and only if the ratio $\delta=R_{o} / R_{c}$ equals $1 / \sqrt{3}$ or $2 / \sqrt{3}$. (See [4].) This means that there exist two different irreducible 14-velocity models. (Here the similar compound models are regarded as identical.) It is shown that the dimension of the space of collision invariants is five for either cases.

Next we give a review of the 32 -velocity model. We take a dodecahedron centered at the origin of the velocity space. The twenty vertices of the dodecahedron defines an element model $M_{d}$. The centers of twelve surfaces of the dodecahedron form an icosahedron and define another element model $M_{i}$. Let $\mathscr{F}_{d}$ and $\mathscr{F}_{i}$ be the families of discrete models similar to $M_{d}$ and $M_{i}$, respectively. Let us consider a compound model of these two kinds of element models. We denote the moduli of the velocities of two element models by $R_{d}$ and $R_{i}$, respectively. In other words, $R_{d}$ and $R_{i}$ are the radii of the circumspheres of the dodecahedron and the icosahedron corresponding to these models. Then the mixed collisions exist if and only if the ratio $\gamma=R_{i} / R_{d}$ equals one of the four values:

$$
\sqrt{(5 \pm \sqrt{5}) / 6}, \quad \sqrt{(5 \pm 2 \sqrt{5}) / 3}
$$

This fact was noticed by Cabannes [3], [4]. Therefore, we have four different irreducible 32-velocity models provided that similar compound models are identified.

In order to check the stability condition for the 32-velocity models, we need a suitable choice of the cartesian coordinate system. Besides the geometric description of the 32-velocity models, Cabannes gave concrete coordinates to the velocities [3], [4]. But this cartesian coordinate system seems to be ill-suited for our purpose. The appropriate choice of the cartesian coordinate system, which will be given in $\S 4$, enables us to express each of the 32 velocities by a sextuple of rational integers. This means that the problem of the verification of the stability condition for the four 32-velocity models becomes algorithmically solvable by this choice of the cartesian coordinate system. We give here the definition of the equivalence between 
discrete models. Two discrete models are said to be equivalent if one is similar to the other after the action of a suitable orthogonal transformation. We may say therefore that we study the 32-velocity models not in its original presentation by Cabannes but in its equivalent form.

Finally we point out the fact that the dimension of the space of collision invariants is eight for the 32 -velocity models. This is the striking contrast between the 14 - and 32-velocity models. In spite of the strange appearance of this fact, these models are normal in the sense that any collision invariant is expressed as a linear combination of eight particular collision invariants issuing from the conservation of mass, momentum, and energy.

The contents of this paper is as follows. In $\S 2$, we give a survey of the general results on discrete models obtained in [8], [9]. We introduced in [10], [11] the definition of the regularity for discrete velocity models of the Boltzmann equation. This will be summarized in $\S 3$ for later use. It is shown in $\S 4$ that the $32-$ velocity models are regular and hence the results of [8], [9] are applied to these models. Our computation follows the lines of [9] except for the use of the computer. Some of the results of computations are given in Appendices 2, 3, 4, and 5.

We note that Cercignani [5] formulated a criterion for checking the stability condition under the hypothesis that the dimension of the space of collision invariants equals $n+2$. Here $n$ is the dimension of the space in which the discrete model is considered. (Hence $n+2=5$ in case of $n=3$.) We shall give in Appendix 1 a generalization of this criterion to the cases where the dimension of the space of collision invariants equals either $2 n+2$ or $2 n+3$. (Note that $2 n+2=8$ and $2 n$ $+3=9$ when $n=3$.) The regularity of the 32 -velocity models can be verified also by using this criterion.

We would like to thank Professor H. Cabannes for sending one of us a list of his computer program for writing down the whole system of equations of the 32 -velocity model $\left(\gamma=\gamma_{1}\right)$. Although we chose a different approach to the problem, we were much stimulated by his work. We thank him also for sending us a preprint of his paper [4]. 


\section{§2. General Theory of Discrete Models}

In this section we give a brief summary of the results on the discrete velocity models of the Boltzmann equation obtained in [8], [9]. In what follows, we say simply the discrete models in place of the discrete velocity models.

Let $u_{1}, \ldots, u_{m}$ be a set of distinct vectors in the velocity space $\boldsymbol{R}^{n}$. By a vector we mean a directed line segment starting from the origin of the velocity space. Since each vector determines, and is determined by, its end-point, it is usual to identify a vector with its end-point. Thus we may regard $u_{1}, \ldots, u_{m}$ as a finite set of points of the velocity space $\mathbb{R}^{n}$. Let $F_{i}=F_{i}(t, x)$ be the density distribution function for the molecules with velocity $u_{i}$ at time $t$ and position $x=\left(x_{1}, \ldots, x_{n}\right) \in \mathbb{R}^{n}$. The following system of equations describing the evolution of the gas is called the discrete Boltzmann equation:

$$
\frac{\partial F_{i}}{\partial t}+u_{i} \cdot \nabla_{x} F_{i}=Q_{i}(F, F), \quad i=1, \ldots, m .
$$

Here $\nabla_{x} F_{i}=\left(\partial F_{i} / \partial x_{1}, \ldots, \partial F_{i} / \partial x_{n}\right)$, and $u_{i} \cdot \nabla_{x} F_{i}$ denotes the standard inner-product of the $n$-dimensional vectors $u_{i}$ and $\nabla_{x} F_{i}$. The collision term $Q_{i}(F, F)$ is given by

$$
Q_{i}(F, F)=\frac{1}{2} \sum_{j, k, l}\left(A_{k l}^{i j} F_{k} F_{l}-A_{i j}^{k l} F_{i} F_{j}\right), \quad i=1, \ldots, m,
$$

where the coefficients $A_{i j}^{k l}, 1 \leq i, j, k, l \leq m$, are non-negative constants related to the transition probability of the binary collision with initial state $\left(u_{i}, u_{j}\right)$ and final state $\left(u_{k}, u_{l}\right) .\left(A_{i j}^{k l}\right.$ is set to be zero for all non-realizable collisions. Otherwise, $A_{i j}^{k l}$ is a positive constant.) We assume that

$$
A_{i j}^{k l}=A_{j i}^{k l}=A_{i j}^{l k}
$$

and that

$$
A_{i j}^{k l}=A_{k l}^{i j}
$$

for any $i, j, k, l$. The latter condition $(2.3)_{2}$ is called the microreversibility. We assume furthermore the existence of collision. This means that

$$
A_{i j}^{k l} \neq 0 \text { for some } i, j, k, l \text {. }
$$

Now we write $(2.1)$ in another form. Let $u_{i}=\left(u_{i}^{1}, \ldots, u_{i}^{n}\right), i=1, \ldots$, 
$m$, and let

$$
U^{j}=\operatorname{diag}\left(u_{1}^{j}, \ldots, u_{m}^{j}\right), \quad j=1, \ldots, n 。
$$

Then (2.1) can be rewritten as

$$
F_{t}+\sum_{j=1}^{n} U^{j} F_{x_{j}}=Q(F, F)
$$

where $F={ }^{t}\left(F_{1}, \ldots, F_{m}\right), \quad Q(F, F)={ }^{t}\left(Q_{1}(F, F), \ldots, Q_{m}(F, F)\right), \quad F_{t}=$ $\partial F / \partial t, F_{x_{j}}=\partial F / \partial x_{j}$ 。

We recall here two basic notions in the discrete kinetic theory. One is collision invariant and the other is Maxwellian. An element $\phi={ }^{t}\left(\phi_{1}, \ldots, \phi_{m}\right)$ of $\mathbb{R}^{m}$ is called a collision invariant if $\phi$ satisfies

$$
A_{i j}^{k l}\left(\phi_{i}+\phi_{j}-\phi_{k}-\phi_{l}\right)=0
$$

for any $i, j, k, l$. The set of all collision invariants forms a subspace of $\mathbb{R}^{m}$ and is denoted by $\mathscr{M}$. Since ${ }^{t}(1, \ldots, 1) \in \mathscr{M}$, it follows from (2.4) that $0<\operatorname{dim} \mathscr{M}<m$. Now let $N={ }^{t}\left(N_{1}, \ldots, N_{m}\right) \in \mathbb{R}^{m}$ and let $N_{i}>0$ for $i=1, \ldots, m$. Then $N$ is said to be a Maxwellian if it satisfies

$$
A_{i j}^{k l}\left(N_{i} N_{j}-N_{k} N_{l}\right)=0,
$$

for any $i, j, k, l$. This is equivalent to saying that $\log N={ }^{t}\left(\log N_{1}, \ldots\right.$, $\log N_{m}$ ) is a collision invariant. A function $F$ of time $t$ and position $x$ with values in the set of Maxwellians is called a locally Maxwellian. A constant Maxwellian $N$ may also be regarded as a function of time $t$ and position $x$. In this case, $N$ is called an absolute Maxwellian. It is well-known that $N$ is a Maxwellian if and only if $Q(N, N)=0$. (Here, $N>0$, namely, $N_{i}>0$ for $i=1, \ldots, m$, is assumed.) Hence an absolute Maxwellian is a constant stationary solution of (2.1).

Finally we state for later use one of the conditions obtained in [9], which guarantees the global existence of solutions to (2.1) and their asymptotic approach to the absolute Maxwellian. To this end, we introduce the following notation,

$$
U(\omega)=\sum_{j=1}^{n} U^{j} \omega_{j}, \quad \omega=\left(\omega_{1}, \ldots, \omega_{n}\right) \in S^{n-1} 。
$$

Then we have

(2.10) Stability condition: Let $\phi \in \mathscr{M}$ and let $\mu \phi+U(\omega) \phi=0$ for some $\mu \in \mathbb{R}$ and $\omega \in S^{n-1}$. Then $\phi=0$.

We refer the reader to [9] for the other equivalent conditions and 
for the precise statements of the global existence theorem. (See Theorem 5. 1. of [9].)

\section{§ 3. Regular Discrete Models}

In the study of discrete models [10], [11], we singled out a category which we called the regular discrete models. One of the basic examples is the 14-velocity model. It will become clear at the end of this paper that the 32-velocity model is another important example. We give in this section a review of regular discrete models.

Let $u_{1}, \ldots, u_{m}$ be distinct vectors in $\boldsymbol{R}^{n}$. We set $M=\left\{u_{1}, \ldots, u_{m}\right\}$ and assume that the linear span of $M$ coincides with $\boldsymbol{R}^{n}$. First we give a general recipe for determing the constants $A_{i j}^{k l}$ that appear in the collision term $Q(F, F)$ of the discrete Boltzmann equation (2.1). For this purpose, we recall the definition of collision. We define $\Sigma$ to be the set of all unordered pairs of distinct velocities. The binary collision means simply a relation on $\Sigma$ which will be specified below. We set for simplicity

$$
\Sigma=\left\{\left(u_{i}, u_{j}\right) ; 1 \leq i<j \leq m\right\}
$$

by using the lexicographical order. Let $\alpha, \beta \in \Sigma$. Then, $\alpha=\left(u_{i}, u_{j}\right)$, $\beta=\left(u_{k}, u_{l}\right)$ for some $i, j, k, l$ where $1 \leq i<j \leq m, 1 \leq k<l \leq m$. We write temporalily $(\alpha, \beta)$ for the ordered couple of $\alpha$ and $\beta$. Then $(\alpha, \beta)$ is called a collision if it satisfies the following properties:

(3. 2) $)_{1} \quad \alpha \neq \beta$.

$(3.2)_{2}$ The momentum of $\alpha$ equals the momentum of $\beta$, i. e., $u_{i}+u_{j}=u_{k}+u_{l}$.

(3.2) ${ }_{3}$ The energy of $\alpha$ equals the energy of $\beta$, i. e., $\left|u_{i}\right|^{2}+\left|u_{j}\right|^{2}=\left|u_{k}\right|^{2}+\left|u_{l}\right|^{2}$.

It is customary to denote the collision $(\alpha, \beta)$ by $\alpha \rightarrow \beta$ and $\alpha, \beta$ are called the initial and the final states, respectively. If $\alpha \rightarrow \beta$ is a collision, then $\beta \rightarrow \alpha$ is also a collision and vice versa. The collision $\beta \rightarrow \alpha$ is usually referred to as the restitution collision of the original collision $\alpha \rightarrow \beta$. We denote by $\mathscr{C}$ the totality of collisions and assume that $\mathscr{C}$ is not an empty set. Let $G$ be the transformation group associated with the discrete model $M$. (Note thet $G$ is determined uniquely by 
$M$ as the set of all isometric transformations on $M$. In particular $G$ is a finite group.) We introduce an equivalence relation on $\mathscr{C}$ by means of $G$. Let $g \in G$ and let $\alpha=\left(u_{i}, u_{j}\right) \in \Sigma$. We define $g \alpha$ to be the unordered pair $\left(g u_{i}, g u_{j}\right)$. Two collisions $\alpha \rightarrow \beta$ and $\alpha^{\prime} \rightarrow \beta^{\prime}$ are said to be equivalent if one of the following two conditions holds:

(3.3) ${ }_{1} \quad \alpha^{\prime} \rightarrow \beta^{\prime}$ equals $g \alpha \rightarrow g \beta$ for some $g \in G$.

(3.3) ${ }_{2} \quad \alpha^{\prime} \rightarrow \beta^{\prime}$ equals $g \beta \rightarrow g \alpha$ for some $g \in G$.

It is easily seen that this is an equivalence relation on $\mathscr{C}$. Hence we have a partition of $\mathscr{C}$ by this equivalence relation. Let $C_{1}, \ldots, C_{q}$ be the set of equivalence classes. Then the constants $A_{i j}^{k l}$ are defined by

$$
A_{i j}^{k l}=\left\{\begin{array}{l}
a_{s}, \text { if }\left(u_{i}, u_{j}\right) \rightarrow\left(u_{k}, u_{l}\right) \text { is a collision of } C_{s},(1 \leq s \leq q), \\
0, \text { if the ordered pair of }\left(u_{i}, u_{j}\right) \text { and }\left(u_{k}, u_{l}\right) \text { is not a } \\
\text { collision. }
\end{array}\right.
$$

Here $a_{1}, \ldots, a_{q}$ are arbitrary positive constants. It is to be noted that the definitions of collision invariants and Maxwellians do not depend on the particular choice of the $a_{s}$ 's.

Lemma 3. 1. Let $M=\left\{u_{1}, \ldots, u_{m}\right\}$ be a given discrete model. We assume that the linear span of $M$ coincides with $\mathbb{R}^{n}$ and that there exists at least one collision. We assume furthermore the constants $A_{i j}^{k l}, \quad 1 \leq$ $i, j, k, l \leq m$, are determined by (3.4). Then

(i) $(2.3)_{1}$ and $(2.3)_{2}$ hold true.

(ii) the collision term $Q(F, F)$ defined by $(2.2)$ is invariant under the transformation group $G$. Namely, for any $g \in G$ and $F \in \mathbb{R}^{m}$, we have $g Q(F, F)=Q(g F, g F)$. Here $F$ is regarded as a function defined on $M$. More precisely, $(g F)_{i}=F_{o(i)}$ where $\sigma=\rho(g)$, $\rho$ being faithful permutation representation of $G$.

Finally we give the definition of regular discrete models introduced in [10], [11].

Definition 3.1. The discrete model described by the equations $(2.1)$ is said to be regular if the following three conditions are satisfied:

$(3.5)_{1}$ The equations (2.1) is irreducible in the sense that it does not 
split into two decoupled subsystems.

$(3.5)_{2}$ The collision term $Q(F, F)$ is invariant under $G$ in the sense specified in Lemma 3. 1.

$(3.5)_{3}$ The stability condition (2.10) holds true.

It should be noted that, by the definition of $A_{i j}^{k l}$ (see (3.4)), $(3.5)_{2}$ is automatically satisfied. We point out also that, by $(3.5)_{3}$, the global existence theorem in [9] can be applied to any regular discrete model.

\section{§4. 32-Velocity Models}

\section{Velocity Vectors}

We consider a dodecahedron of edge of length 2 with center at the origin of the velocity space. It is well-known that there exist five inscribed cubes. Each vertex of the dodecahedron belongs to two of the cubes. The length of edge of these cubes are $2 \tau$, where $\tau=(1+\sqrt{5}) / 2$. Note that the ratio $1: \tau$ is known as golden section and that $\tau^{2}=\tau+1$. We look at one of the five inscribed cubes and we let coincide the three axes of 4 -fold rotation of the cube with the $x$-, $y$-, and $z$-axes of the cartesian coordinate system. Thus the centers of six surfaces of the cube are lying on one of the $x^{-}, y^{-}$, and $z$-axes. The twenty vertices of the dodecahedron are given by

$$
\begin{aligned}
& ( \pm 1,0, \pm(1+\tau)),(0, \pm(1+\tau), \pm 1) \\
& ( \pm(1+\tau), \pm 1,0),( \pm \tau, \pm \tau, \pm \tau) .
\end{aligned}
$$

Let the vectors starting from the origin and ending at these vertices be $v_{i}^{(1)}, 1 \leq i \leq 20$. A simple computation shows that the centers of twelve surfaces of the dodecahedron are expressed as

$$
\begin{aligned}
& \frac{1}{5}(0, \pm(1+3 \tau), \pm(3+4 \tau)), \frac{1}{5}( \pm(1+3 \tau), \pm(3+4 \tau), 0), \\
& \frac{1}{5}( \pm(3+4 \tau), 0, \pm(1+3 \tau))
\end{aligned}
$$

by the same cartesian coordinate system. These twelve centers are the vertices of an icosahedron that is in a dual position to the dodecahedron. Let the vectors starting from the origin and ending 
at these vertices be $v_{i}^{(2)}, 1 \leq i \leq 12$. We set

$$
u_{i}=v_{i}^{(1)}(1 \leq i \leq 20), \quad u_{i}=\delta v_{1-20}^{(2)} \quad(21 \leq i \leq 32) .
$$

Here $\delta$ is a positive constant which will be specified below. We define $M^{(1)}, M^{(2)}$, and $M$ as follows.

$$
\begin{aligned}
& M^{(1)}=\left\{u_{i} ; 1 \leq i \leq 20\right\}, \quad M^{(2)}=\left\{u_{i} ; 21 \leq i \leq 32\right\}, \\
& M=\left\{u_{i} ; 1 \leq i \leq 32\right\}=M^{(1)} \cup M^{(2)} .
\end{aligned}
$$

$M^{(1)}$ and $M^{(2)}$ are the 20- and 12-velocity models of Cabannes [2]. Noting that $M^{(1)}$ and $M^{(2)}$ are element models, we set

$$
R_{1}=\left|u_{i}\right| \quad(1 \leq i \leq 20), \quad R_{2}=\left|u_{i}\right| \quad(21 \leq i \leq 32) .
$$

Then we have $R_{1}=R_{d}, R_{2}=\delta r_{d}$, if the respective radii of the circumscribing and inscribing spheres of the dodecahedron are denoted by $R_{d}$ and $r_{d}$. Since it is known that

$$
r_{d} / R_{d}=\sqrt{(5+2 \sqrt{5}) / 15}
$$

we obtain

$$
\gamma \equiv R_{2} / R_{1}=\delta \sqrt{(5+2 \sqrt{5}) / 15}
$$

Now we introduce notations for collisions. We denote by $\mathscr{C}$ the totality of collisions of the 32-velocity model $M$. The sets of collisions of the models $M^{(1)}$ and $M^{(2)}$ are denoted by $\mathscr{C}^{(1)}$ and $\mathscr{C}^{(2)}$, respectively. We observe that, if the initial state of a collision consists of an element of $M^{(1)}$ and an element of $M^{(2)}$, the same is true for the final state. Such collisions are called mixed collisions. We denote by $\mathscr{C}^{(1,2)}$ the set of mixed collisions of the 32-velocity model $M$. Clearly we have

$$
\mathscr{C}=\mathscr{C}^{(1)} \cup \mathscr{C}^{(2)} \cup \mathscr{C}^{(1,2)}
$$

The following result concerning the existence of mixed collisions was obtained by Cabannes [3], [4].

Lemma 4. 1. $\mathscr{C}^{(1,2)}$ is not an empty set if and only if the value of $\gamma$ defined by (4.7) equals with one of the following number,

$$
\begin{aligned}
& \gamma_{1}=\sqrt{(5-\sqrt{5}) / 6}, \quad \gamma_{2}=\sqrt{(5+\sqrt{5}) / 6}, \\
& \gamma_{3}=\sqrt{(5-2 \sqrt{5}) / 3}, \quad \gamma_{4}=\sqrt{(5+2 \sqrt{5}) / 3} .
\end{aligned}
$$

Proof. We give a sketch of the proof based on the coordinates (4. 1), (4.2). The computation can be carried through without using 
the computer. $\mathscr{C}^{(1,2)}$ is not an empty set if and only if there exist $v_{i}^{(1)}, v_{j}^{(1)}, v_{k}^{(2)}, v_{l}^{(2)}(1 \leq i \neq j \leq 20,1 \leq k \neq l \leq 12)$ such that

$$
v_{i}^{(1)}-v_{j}^{(1)}=\delta\left(v_{k}^{(2)}-v_{l}^{(2)}\right) \text {. }
$$

We make up the complete lists of $\left\{v_{i}^{(1)}-v_{j}^{(1)} ; i \neq j\right\}$ and $\left\{v_{k}^{(2)}-v_{l}^{(2)} ; k \neq l\right\}$. Then it turns out that $\delta$ coincides with one of the following numbers,

$$
\begin{aligned}
& \delta_{1}=-4+3 \tau=(-5+3 \sqrt{5}) / 2, \quad \delta_{2}=3-\tau=(5-\sqrt{5}) / 2, \\
& \delta_{3}=7-4 \tau=5-2 \sqrt{5}, \quad \delta_{4}=-1+2 \tau=\sqrt{5}
\end{aligned}
$$

if the mixed collisions exist. Here the sign of $\delta$ is neglected. This combined with (4.7) gives (4.9).

As a consequence, the 12 velocity vectors $u_{i}, 21 \leq i \leq 32$, are determined as follows according to the respective values of $\gamma$.

$(4.12)_{1}(0, \pm 1, \pm \tau),( \pm 1, \pm \tau, 0),( \pm \tau, 0, \pm 1)$,

$(4.12)_{2} \quad(0, \pm \tau, \pm(1+\tau)),( \pm \tau, \pm(1+\tau), 0),( \pm(1+\tau), 0, \pm \tau)$,

$(4.12)_{3}(0, \pm(-1+\tau), \pm 1),( \pm(-1 \pm \tau), \pm 1,0),( \pm 1,0, \pm(-1+\tau))$,

$(4.12)_{4} \quad(0, \pm(1+\tau), \pm(1+2 \tau)),( \pm(1+\tau), \pm(1+2 \tau), 0)$,

$$
( \pm(1+2 \tau), 0, \pm(1+\tau)) \text {. }
$$

The 20 velocity vectors $u_{i}, 1 \leq i \leq 20$, are given by the common coordinates (4.1) for $\gamma=\gamma_{1}, \ldots, \gamma_{4}$. It should be emphasized that all the coordinates are algebraic integers of $\boldsymbol{Q}(\sqrt{5})$, the rationals adjoined by $\sqrt{5}$. In these situations we can restrict our computation to the integral arithmetic. Note that the standard integral basis of $\boldsymbol{Q}(\sqrt{5})$ is $\{1, \tau\}$.

\section{Collisions}

The number of collisions for the 32-velocity models is given Cabannes [4]. The results, obtained independently by us, agrees with that of Cabannes.

Lemma 4.2. Let $p$ be the number of the totality of collisions. Let $p^{(1)}, p^{(2)}$, and $p^{(1,2)}$ be the respective numbers of collisions contained in $\mathscr{C}^{(1)}$, $\mathscr{C}^{(2)}$, and $\mathscr{C}^{(1,2)}$. Then, in the case of $\gamma=\gamma_{1}$ or $\gamma_{2}$, we have $p=540$. When $\gamma=\gamma_{3}$ or $\gamma_{4}$, we have $p=300$. In all cases, $p^{(1)}=150$ and $p^{(2)}=30$. We have $p^{(1,2)}=360$ for $\gamma=\gamma_{1}, \gamma_{2}$ and $p^{(1,2)}=120$ for $\gamma=\gamma_{3}, \gamma_{4}$. 
The complete lists of collision are omitted here. Next we turn to the classification of collisions according to the procedure given in $\S 2$. The transformation group $G$, namely, the symmetry group of $M=\left\{u_{1}, \ldots, u_{32}\right\}$ is used for this purpose. We have $G=A_{5} \times I$, where $I$ denotes the group of order 2 generated by the inversion in the center of symmetry. The notation is that of Coxeter [6]. The following result, as well as the result in Lemma 4.2 , is obtained by using the computer.

Lemma 4. 3. Let $q$ be the number of equivalence classes contained in $\mathscr{C}$. Let $q^{(1)}, q^{(2)}, q^{(1,2)}$ be the number of equivalence classes contained in $\mathscr{C}^{(1)}, \mathscr{C}^{(2)}$, and $\mathscr{C}^{(1,2)}$, respectively. Then, we have $q=7$ for $\gamma=\gamma_{1}, \gamma_{2}$ and $q=6$ for $\gamma=\gamma_{3}, \gamma_{4}$. In all cases, $q^{(1)}=3$ and $q^{(2)}=1$. More precisely, $\mathscr{C}^{(1)}$ consists of three equivalence classes and two of them contain 60 collisions. The rest contains 30 collisions. $\mathscr{C}^{(2)}$ consists of only one equivalence class containing 30 collisions. When $\gamma=\gamma_{1}$ or $\gamma_{2}$, we have $q^{(1,2)}=3$. In this case, $\mathscr{C}^{(1,2)}$ consists of three equivalence classes each of which contains 120 collisions. When $\gamma=\gamma_{3}$ or $\gamma_{4}$, we have $q^{(1,2)}=2$. In this case, $\mathscr{C}^{(1,2)}$ consists of two equivalence classes each of which contains 60 collisions.

Now we look at the classification of collisions from different viewpoint. For each collision $\left(u_{i}, u_{j}\right) \rightarrow\left(u_{k}, u_{l}\right)$, we set

$$
\begin{aligned}
& E_{i j}^{k l}=\left|u_{i}\right|^{2}+\left|u_{j}\right|^{2}=\left|u_{k}\right|^{2}+\left|u_{l}\right|^{2}, \\
& M_{i j}^{k l}=\left|u_{i}+u_{j}\right|=\left|u_{k}+u_{l}\right|, \\
& I_{i j}^{k l}=\left|\left(u_{i}-u_{j}\right) \cdot\left(u_{k}-u_{l}\right)\right| /\left|u_{i}-u_{j}\right| \cdot\left|u_{k}-u_{l}\right| .
\end{aligned}
$$

Here $E_{i j}^{k l}$ and $M_{i j}^{k l}$ represent total energy and the modulus of total momentum, respectively. $I_{i j}^{k l}$ equals $|\cos \theta|$, where $\theta$ denotes the angle of deflection in the center of mass system. Each of $E_{i j}^{k l}, M_{i j}^{k l}$, and $I_{i j}^{k l}$ takes a constant value on any equivalence class. But at least one of the three quantities is different for distinct equivalence classes. This can be checked by the computer. We may say therefore that $E_{i j}^{k l}, M_{i j}^{k l}$, and $I_{i j}^{k l}$ form a complete set of labels. The details will be given in Appendix 3. It is to be noted that Cabannes [4] used only $E_{i j}^{k l}$ and $M_{i j}^{k l}$. Finally we remark that the collision term $Q_{i}(F, F), i=1, \ldots, 32$, can be written down once the enumeration of collisions is finished. We shall give the concrete forms of $Q_{1}(F, F)$ and $Q_{21}(F, F)$ for $\gamma=\gamma_{1}$ and $\gamma=\gamma_{3}$ in Appendix 4 . 


\section{Collision Invariants}

We want to determine the space $\mathscr{M}$ of collision invariants for the 32-velocity models. It is shown that $\mathscr{M}$ has dimension 8 for $\gamma=$ $\gamma_{1}, \ldots, \gamma_{4}$. The result may also be checked without using the computer. We write $u_{i}=\left(u_{i}^{1}, u_{i}^{2}, u_{i}^{3}\right)$ for $i=1, \ldots, 32$. It is a known fact that the following five vectors

$$
\begin{aligned}
& \phi^{(0)}={ }^{t}(1, \ldots, 1), \\
& \phi^{(j)}={ }^{t}\left(u_{1}^{j}, \ldots, u_{32}^{j}\right), \\
& \phi^{(4)}={ }^{t}\left(\left|u_{1}\right|^{2}, \ldots,\left|u_{32}\right|^{2}\right), \quad j=1,2,3,
\end{aligned}
$$

are collision invariants. We observe that each $\phi^{(i)}, 0 \leq i \leq 4$, is a $32-$ tuple of algebraic integers of $\boldsymbol{Q}(\sqrt{5})$. By means of the standard integral basis $\{1, \tau\}$ of $\boldsymbol{Q}(\sqrt{5})$, we set

$$
u_{i}=v_{i}+\tau w_{i}, \quad v_{i}, w_{i} \in \mathbb{Z}^{3},
$$

for $i=1, \ldots, 32$. Let $v_{i}=\left(v_{i}^{1}, v_{i}^{2}, v_{i}^{3}\right)$ and let $w_{i}=\left(w_{i}^{1}, w_{i}^{2}, w_{i}^{3}\right)$, We set

$$
\begin{aligned}
& \psi^{(j)}={ }^{t}\left(v_{1}^{j}, \ldots, v_{32}^{j}\right), \\
& \phi^{(3+j)}={ }^{t}\left(w_{1}^{j}, \ldots, w_{32}^{j}\right), \quad j=1,2,3, \\
& \phi^{(7)}={ }^{t}\left(\left|v_{1}\right|^{2}+\left|w_{1}\right|^{2}, \ldots,\left|v_{32}\right|^{2}+\left|w_{32}\right|^{2}\right), \\
& \phi^{(8)}={ }^{t}\left(2 v_{1} \cdot w_{1}+\left|w_{1}\right|^{2}, \ldots, 2 v_{32} \cdot w_{32}+\left|w_{32}\right|^{2}\right) \text {. }
\end{aligned}
$$

Then

$$
\begin{aligned}
& \phi^{(k)}=\psi^{(k)}+\tau \psi^{(3+k)}, \quad k=1,2,3, \\
& \phi^{(4)}=\psi^{(7)}+\tau \psi^{(8)} .
\end{aligned}
$$

It is easily seen that the vectors $\psi^{(1)}, \ldots, \psi^{(6)}$ are lineary independent for $\gamma=\gamma_{1}, \ldots, \gamma_{4}$. The vectors $\phi^{(0)}, \phi^{(7)}, \phi^{(8)}$ are linearly dependent but two of these vectors are linearly independent for all cases.

Lemma 4. 4. Let $\gamma=\gamma_{1}, \ldots, \gamma_{4}$. Then the space $\mathscr{M}$ of collision invariants has dimension 8. A basis of $\mathscr{M}$ is given by six vectors $\psi^{(1)}, \ldots, \phi^{(6)}$ supplemented by two vectors arbitrarily chosen from $\phi^{(0)}, \psi^{(7)}, \psi^{(8)}$.

Remark. Let $\chi^{(1)}$ be the vector whose $i$-th component equals 1 for $i=1, \ldots, 20$ and equals 0 for $i=21, \ldots, 32$. Let $\chi^{(2)}=\phi^{(0)}-\chi^{(1)}$. Then $\chi^{(1)}$ and $\chi^{(2)}$ are linearly independent and both vectors belong to $\mathscr{M}$. Moreover the linear span of $\left\{\chi^{(1)}, \chi^{(2)}\right\}$ equals the linear span 
of $\left\{\phi^{(0)}, \phi^{(7)}, \phi^{(8)}\right\}$. Hence $\phi^{(1)}, \ldots, \phi^{(6)}, \chi^{(1)}, \chi^{(2)}$ form a basis of $\mathscr{M}$. It is this basis that is used in the actual computation. In any case, all the collision invariants issue from the conservation of mass, momentum and energy in the 32-velocity models.

\section{4. $\quad \mathbb{P}$-Sets and the Stability Condition}

For the reason that will become clear soon, we introduce here the definition of $P$-sets. Let $J$ be a non-empty subset of $\{1,2, \ldots$, 32\}. Then $J$ is called a $P$-set if there exists a plane $\Pi$ satisfying the following properties: $u_{j} \in M$ lies on $\Pi$ if and only if $j \in J$. The totality of $P$-sets will be denoted by $\mathscr{P}$. Any $P$-set, which is contained in another $P$-set as a proper subset, is called a $P$-set of the second category. All other $P$-sets are of the first category. We denote by $\mathscr{Q}$ and $\mathscr{R}$ the totalities of the $P$-sets of the first and the second categories, respectively. By definition $\mathscr{Q}$ and $\mathscr{R}$ are disjoint and $\mathscr{P}=\mathscr{Q} \cup \mathscr{R}$. Now we assign to each $P$-set a subspace of $\mathbb{R}^{32}$. Let $J \in \mathscr{P}$. We define $\mathscr{N}_{J}$ to be the subspace of $\mathbb{R}^{32}$ spaned by $\left\{e_{j} ; j \in J\right\}$. Here $\left\{e_{j} ; 1 \leq j \leq 32\right\}$ denotes the standard basis of $\mathbb{R}^{32}$. Namely, the $i$-th component of $e_{j}$ equals $\delta_{i j}$. We rewrite the stability condition (2.10) by means of $P$-sets.

Stability condition reformulated: Let $\mathscr{M}$ be the space of collision invariants and let $J \in \mathscr{P}$. Let $\mathscr{N}_{J}$ be the subspace of $\mathbb{R}^{32}$ defined above. Then

$$
\mathscr{M} \cap \mathscr{N}_{J}=\{0\}, \quad \text { for any } J \in \mathscr{P} \text {. }
$$

To see the equivalence of two statements, we suppose that $\phi \in \mathscr{M}$ and that $\mu \phi+U(\omega) \phi=0$ for some $\mu \in \mathbb{R}$ and $\omega \in S^{2}$. Since $U(\omega)=$ $\operatorname{diag}\left(u_{1} \cdot \omega, \ldots, u_{32} \cdot \omega\right)$, the set of all $j$ such that $-\mu=u_{j}{ }^{\circ} \omega$ forms a $P$-set $J$. Then $\phi=0$ follows from $\mathscr{M} \cap \mathcal{N}_{J}=\{0\}$. The converse assersion holds true also. It is easy to see that in the stability condition reformulated we may replace $\mathscr{P}$ by 2 . Therefore we have little interest in the $P$-sets of the second category. We set

$$
\mathscr{Q}^{(i)}=\{J \in \mathscr{Q} ;|J|=i\}, \quad i=1, \ldots, 32,
$$

where $|J|$ denotes the cardinality of $J$. 
Lemma 4.5. Let $r$ be the number of P-sets of the first category. Let $r^{(i)}$ be the number of $P$-sets contained in $\mathscr{Q}^{(i)}$, where $i=1, \ldots, 32$. Then, in the case of $\gamma=\gamma_{1}$ or $\gamma_{2}$, we have $r=1781$. When $\gamma=\gamma_{3}$ or $\gamma_{4}$ we have $r=759$. More precisely, if $\gamma=\gamma_{1}, \gamma_{2}$, then $r^{(3)}, r^{(4)}, r^{(5)}, r^{(6)}$, and $r^{(8)}$ are 1320, 360, 36, 50, and 15, respectively. Other $r^{(i)}$ 's are zero. If $\gamma=\gamma_{3}, \gamma_{4}$, then $r^{(3)}, r^{(4)}, r^{(5)}, r^{(8)}, r^{(9)}$, and $r^{(10)}$ are 520,120, 72, 15, 20, and 12, respectively. Other $r^{(i)}$ s are zero.

Now we give a classification of $P$-sets by the symmetry group $G=A_{5} \times I$ associated with $M=\left\{u_{1}, \ldots, u_{32}\right\}$. Two $P$-sets are regarded as equivalent if one is obtained from the other by the action of a suitable symmetry transformation. Thus we have a partition of $\mathscr{P}$. Accordingly, $\mathscr{Q}$ and each $\mathscr{Q}^{(i)} 1 \leq i \leq 32$, are classified also.

Lemma 4.6. Let $s$ and $s^{(i)}$ be the respective numbers of equivalence classes contained in $\mathscr{Q}$ and $\mathscr{Q}^{(i)}$, where $i=1, \ldots, 32$. Then, in the case of $\gamma=\gamma_{1}$ or $\gamma_{2}$, we have $s=32$. When $\gamma=\gamma_{3}$ or $\gamma_{4}$, we have $s=17$. More precisely, if $\gamma=\gamma_{1}, \gamma_{2}$, then $s^{(3)}, s^{(4)}, s^{(5)}, s^{(6)}$, and $s^{(8)}$ are $19,7,3,2$, and 1 , respectively. If $\gamma=\gamma_{3}, \gamma_{4}$, then $s^{(3)}, s^{(4)}, s^{(5)}, s^{(8)}, s^{(9)}$, and $s^{(10)}$ are 9,3 , $2,1,1$, and 1 , respectively.

A list of representatives for $\gamma=\gamma_{1}, \ldots, \gamma_{4}$ will be given in Appendix 5 . We observe that if $g \in G$, then $g \mathscr{N}_{J_{J}}=\mathscr{N}_{\sigma(J)}$, where $\sigma=\rho(g), \rho$ being faithful permutation representation of $G$. This implies that, if $\mathscr{M} \cap \mathscr{N}_{J}$ $=\{0\}$ is checked for a particular $J \in \mathscr{Q}$, then the same fact holds for any $P$-set which is equivalent to $J$. Note that $\mathscr{M}$ is invariant under the action of $G$, i. e., $g \mathscr{M}=\mathscr{M}$ for any $g \in G$. These observations lead to the following.

Stability condition in the reduced form: Let $s$ be as in Lemma 4. 6. Let $J(k), 1 \leq k \leq s$, be the set of representatives of equivalence classes of $\mathscr{Q}$. Then

$$
\mathscr{M} \cap \mathscr{N}_{J(k)}=0, \quad \text { for } \quad k=1, \ldots, s .
$$

As a basis of $\mathscr{M}$, we take for example $\left\{\psi^{(1)}, \ldots, \phi^{(6)}, \chi^{(1)}, \chi^{(2)}\right\}$. A basis of $\mathscr{N}_{J(k)}$ is $\left\{e_{j} ; j \in J(k)\right\}$. Then the union of these two sets of vectors is linearly independent if and only if $\mathscr{M} \cap \mathscr{N}_{J(k)}=\{0\}$. The linear independence of these vectors is checked by using the computer for $k=$ 
$1, \ldots, s$ and for $\gamma_{1}, \ldots, \gamma_{4}$. Here $s=32$ when $\gamma=\gamma_{1}$ or $\gamma_{2}$ and $s=17$ when $\gamma=\gamma_{3}$ or $\gamma_{4}$. Thus we arrive at the following conclusion.

Theorem 4.7. The 32-velocity model is a regular discrete model if and only if $\gamma$ equals one of $\gamma_{1}, \ldots, \gamma_{4}$.

As a consequence we obtain the following result by applying Theorem 5.1 of [10].

Theorem 4.8. Consider the initial value problem for the 32-velocity model with $\gamma=\gamma_{1}, \ldots, \gamma_{4}$. Let $N$ be an absolute Maxwellian and let $F(0, x)=F_{0}(x)$ be the initial condition. Then we have:

(i) Let $s \geq 2$ and let $F_{0}-N \in H^{s}\left(\boldsymbol{R}^{3}\right)$. It $\left\|F_{0}-N\right\|_{s}$ is small enough, the initial value problem has a unique global solution $F(t, x)$ such that

$$
F-N \in C^{0}\left(0, \infty ; H^{s}\left(\boldsymbol{R}^{3}\right)\right) \cap C^{1}\left(0, \infty ; H^{s-1}\left(\mathbb{R}^{3}\right)\right) .
$$

Furthermore,

$$
\left\|F(t)-N||_{s}^{2}+\int_{0}^{t}\right\| D_{x} F(\tau)\left\|_{s-1}^{2} d \tau \leq C\right\| F_{0}-N \|_{s}^{2}
$$

for any $t \in[0, \infty)$, where $G$ is a constant not depending on $t . F(t, x)$ converges to the absolute Maxwellian $N$ uniformly in $x \in \mathbb{R}^{3}$ as $t \rightarrow \infty$.

(ii) Let $s$ be as in (i) and let $p \in[1,2)$. Let $F_{0}-N \in H^{s}\left(\mathbb{R}^{3}\right)$ $\cap L^{p}\left(\boldsymbol{R}^{3}\right)$. If $\left\|F_{0}-N\right\|_{s, p}$ is small enough (the norm $\left\|_{i}\right\|_{s, p}$ denotes the sum of $H^{s}\left(\boldsymbol{R}^{3}\right)$ - and $L^{p}\left(\boldsymbol{R}^{3}\right)$-norms), the solution obtained in (i) satisfies

$$
\|F(t)-N\|_{s} \leq C(1+t)^{-r}\left\|F_{0}-N\right\|_{s, p},
$$

where $G$ is a constant not depending on $t$, and $\gamma=(3 / 2)(1 / p-1 / 2)$.

\section{Appendix 1}

\section{A criterion for the stability condition}

We consider an $n$-dimensional discrete model defined by the velocity vectors $u_{i}=\left(u_{i}^{1}, \ldots, u_{i}^{n}\right), i=1, \ldots, m$. Cercignani obtained in [5] a criterion for the stability condition under the hypothesis that the space $\mathscr{M}$ of collision invariants has dimension $n+2$ and that the following vectors, 


$$
\begin{aligned}
& \phi^{(0)}={ }^{t}(1, \ldots, 1), \\
& \phi^{(j)}={ }^{t}\left(u_{1}^{j}, \ldots, u_{m}^{j}\right), \quad j=1, \ldots, n, \\
& \phi^{(n+1)}={ }^{t}\left(\left|u_{1}\right|^{2}, \ldots,\left|u_{m}\right|^{2}\right),
\end{aligned}
$$

form a basis of $\mathscr{M}$. We modify his argument and obtain analogous results under different circumstances.

Let $K$ be a real quadratic field and let $\{1, \lambda\}$ be the standard integral basis of $K$. Then we have

$$
\lambda^{2}=a \lambda+b, \quad a, b \in \boldsymbol{Z} .
$$

We assume that $u_{i}^{j} \in \boldsymbol{Z}[\lambda]$ for $i=1, \ldots, m, j=1, \ldots, n$. In other words, the $u_{i}$ 's are $n$-tuples of algebraic integers of $K$. Thus,

$$
u_{i}=v_{i}+\lambda w_{i}, \quad v_{i}, m_{i} \in \mathbb{Z}^{n},
$$

for $i=1, \ldots, m$. We set

$$
\begin{aligned}
& \phi^{(j)}=\psi^{(j)}+\lambda \phi^{(n+j)}, \quad j=1, \ldots, n \\
& \phi^{(n+1)}=\psi^{(2 n+1)}+\lambda \psi^{(2 n+2)},
\end{aligned}
$$

where $\phi^{(j)}, j=1, \ldots, n$, and $\phi^{(n+1)}$ are defined by (A. 1). Note that $\psi^{(k)} \in Z^{m}$ for $k=1, \ldots, 2 n+2$. We study the following two cases.

Case 1. The space $\mathscr{M}$ of collision invariants has dimension $2 n+2$. A basis of $\mathscr{M}$ is given either by the vectors $\phi^{(0)}, \phi^{(1)}, \ldots, \phi^{(2 n)}, \psi^{(2 n+1)}$, or by the vectors $\phi^{(0)}, \psi^{(1)}, \ldots, \psi^{(2 n)}, \psi^{(2 n+2)}$.

Case 2. The space $\mathscr{M}$ of collision invariants has dimension $2 n+3$. A basis of $\mathscr{M}$ is given by the vectors $\phi^{(0)}, \psi^{(1)}, \ldots, \psi^{(2 n)}, \psi^{(2 n+1)}, \psi^{(2 n+2)}$.

For notational convenience, we define a matrix $A$ by means of a set of auxiliary vectors. Let $\xi^{(i)}, \eta^{(j)}, \zeta^{(k)}, 1 \leq i \leq r, 1 \leq j, k \leq s$, be any ordered sets of vectors of $\mathbb{R}^{m}$ and let $r+s \leq m$. The $2 m \times 2(r+s)$ matrix $A$ is defined by

$$
A=\left[\frac{\xi^{(1)}, \ldots, \xi^{(r)}}{0}\left|\frac{0}{\xi^{(1)}, \ldots, \xi^{(r)}}\right| \eta^{(1)}, \ldots, \zeta^{(s)} \mid \frac{b \zeta^{(1)}, \ldots \ldots, b \zeta^{(s)}}{\eta^{(1)}+a \zeta^{(1)}, \ldots, \eta^{(s)}+a_{s^{(s)}}^{(s)}}\right] .
$$

First we treat Case 1. Let $v_{i}=\left(v_{i}^{1}, \ldots, v_{i}^{n}\right)$ and let $w_{i}=\left(w_{i}^{1}, \ldots, w_{i}^{n}\right)$. Then

$$
\begin{aligned}
U^{j} & =\operatorname{diag}\left(u_{1}^{j}, \ldots, u_{m}^{j}\right) \\
& =\operatorname{diag}\left(v_{i}^{j}, \ldots, v_{m}^{j}\right)+\lambda \operatorname{diag}\left(w_{1}^{j}, \ldots, w_{m}^{j}\right)
\end{aligned}
$$

for $1 \leq j \leq n$. We set 


$$
V^{j}=\operatorname{diag}\left(v_{1}^{j}, \ldots, v_{m}^{j}\right), \quad W^{j}=\operatorname{diag}\left(w_{1}^{j}, \ldots, w_{m}^{j}\right) .
$$

List of auxiliary vectors for Case 1.

$\left.1^{\circ}\right) \quad \phi^{(0)}, V^{j} \phi^{(0)} \quad(1 \leq j \leq n), \quad W^{j} \phi^{(0)} \quad(1 \leq j \leq n)$.

$\left.2^{\circ}\right) \quad V^{j} V^{k} \phi^{(0)} \quad(1 \leq j \leq k \leq n), \quad V^{j} W^{k} \phi^{(0)} \quad(1 \leq j, k \leq n)$, $V^{j} \psi^{(2 n+1)}+b W^{j} \phi^{(2 n+2)} \quad(1 \leq j \leq n)$.

$\left.3^{\circ}\right) \quad W^{j} V^{k} \phi^{(0)}(1 \leq j \leq k \leq n), \quad W^{j} W^{k} \phi^{(0)} \quad(1 \leq j, k \leq n)$, $W^{j} \psi^{(2 n+1)}+\left(V^{j}+a W^{j}\right) \psi^{(2 n+2)} \quad(1 \leq j \leq n)$.

Here we understand that the vectors in $2^{\circ}$ ) and $3^{\circ}$ ) with indices $j, k$ are arranged according to the lexicographical order of $(j, k)$.

Proposition A.1. Consider Case 1. Let $r=2 n+1$ and let $s=$ $\left(3 n^{2}+3 n\right) / 2$. Suppose that the rank of matrix $A$ defined by using the sets of vectors listed in $\left.\left.\left.1^{\circ}\right), 2^{\circ}\right), 3^{\circ}\right)$ as the auxiliary vectors $\xi^{(i)}, \eta^{(j)}, \zeta^{(k)}$, equals $3 n^{2}+7 n+2$. Then the stability condition holds true.

Next we turn to Case 2.

List of auxiliary vectors for Case 2.

$\left.1^{\circ}\right) \quad \phi^{(0)}, V^{j} \phi^{(0)}(1 \leq j \leq n), \quad W^{j} \phi^{(0)}(1 \leq j \leq n), \phi^{(2 n+1)}$ 。

$\left.2^{\circ}\right) \quad V^{j} V^{k} \phi^{(0)} \quad(1 \leq j \leq k \leq n), \quad V^{j} W^{k} \phi^{(0)}(1 \leq j, k \leq n)$, $V^{j} \psi^{(2 n+1)}+b W^{j} \phi^{(2 n+2)} \quad(1 \leq j \leq n), \quad V^{j} \psi^{(2 n+1)} \quad(1 \leq j \leq n)$ 。

$\left.3^{\circ}\right) \quad W^{j} V^{k} \phi^{(0)} \quad(1 \leq j \leq k \leq n), \quad W^{j} W^{k} \phi^{(0)} \quad(1 \leq j, k \leq n)$, $W^{j} \psi^{(2 n+1)}+\left(V^{j}+a W^{j}\right) \psi^{(2 n+2)} \quad(1 \leq j \leq n)$, $W^{j} \phi^{(2 n+1)}(1 \leq j \leq n)$.

It is to be understood that the vectors in $2^{\circ}$ ) and $3^{\circ}$ ) with indices $j, k$ are arranged according to the lexicographical order of $(j, k)$.

Proposition A.2. Consider Case 2. Let $r=2 n+2$ and let $s=$ $\left(3 n^{2}+5 n\right) / 2$. Define the matrix $A$ by using the sets of vectors enumerated in $\left.\left.1^{\circ}\right), 2^{\circ}\right), 3^{\circ}$ ) as the auxiliary vectors, $\xi^{(i)}, \eta^{(j)}, \zeta^{(k)}$. Then, if the rank of $A$ equals $3 n^{2}+9 n+4$, the stability condition holds true.

For the proof of these propositions, we use the arguments of Cercignani with suitable modifications. The following observation is also useful: Let $\chi^{(i)}, 1 \leq i \leq k$, be the elements of $\mathbb{K}^{m}$, where $K$ is a real quadratic field. Then, $\chi^{(1)}, \ldots, \chi^{(k)}$ are linearly independent 
over $K$ if and only if these vectors are linearly independent over $\boldsymbol{R}$. Finally we notice that the entries of the matrix $A$ thus defined are all rational integers. This implies that, in computing the rank of $A$, we can avoid the approximate computation.

\section{Appendix 2}

\section{Indexing of velocity vectors}

20 velocities

\begin{tabular}{|c|rrrrrrrrrr|}
\hline & $u_{1}$ & $u_{2}$ & $u_{3}$ & $u_{4}$ & $u_{5}$ & $u_{6}$ & $u_{7}$ & $u_{8}$ & $u_{9}$ & $u_{10}$ \\
\hline$v_{i}^{1}$ & 1 & -1 & -1 & 1 & 0 & 0 & 0 & 0 & 1 & 1 \\
$w_{i}^{1}$ & 0 & 0 & 0 & 0 & 0 & 0 & 0 & 0 & 1 & 1 \\
$v_{i}^{2}$ & 0 & 0 & 0 & 0 & 1 & 1 & -1 & -1 & 1 & -1 \\
$w_{i}^{2}$ & 0 & 0 & 0 & 0 & 1 & 1 & -1 & -1 & 0 & 0 \\
$v_{i}^{3}$ & 1 & 1 & -1 & -1 & 1 & -1 & -1 & 1 & 0 & 0 \\
$w_{i}^{3}$ & 1 & 1 & -1 & -1 & 0 & 0 & 0 & 0 & 0 & 0 \\
\hline \hline & $u_{11}$ & $u_{12}$ & $u_{13}$ & $u_{14}$ & $u_{15}$ & $u_{16}$ & $u_{17}$ & $u_{18}$ & $u_{19}$ & $u_{20}$ \\
\hline$v_{i}^{1}$ & -1 & -1 & 0 & 0 & 0 & 0 & 0 & 0 & 0 & 0 \\
$w_{i}^{1}$ & -1 & -1 & 1 & -1 & -1 & 1 & -1 & 1 & 1 & -1 \\
$v_{i}^{2}$ & -1 & 1 & 0 & 0 & 0 & 0 & 0 & 0 & 0 & 0 \\
$w_{i}^{2}$ & 0 & 0 & 1 & 1 & -1 & -1 & -1 & -1 & 1 & 1 \\
$v_{i}^{3}$ & 0 & 0 & 0 & 0 & 0 & 0 & 0 & 0 & 0 & 0 \\
$w_{i}^{3}$ & 0 & 0 & 1 & 1 & 1 & 1 & -1 & -1 & -1 & -1 \\
\hline
\end{tabular}

12 velocities $\left(\gamma=\gamma_{1}\right)$

\begin{tabular}{|l|rrrrrrrrrrrr|}
\hline & $u_{21}$ & $u_{22}$ & $u_{23}$ & $u_{24}$ & $u_{25}$ & $u_{26}$ & $u_{27}$ & $u_{28}$ & $u_{29}$ & $u_{30}$ & $u_{31}$ & $u_{32}$ \\
\hline$v_{i}^{1}$ & 0 & 0 & 0 & 0 & 1 & -1 & -1 & 1 & 0 & 0 & 0 & 0 \\
$w_{i}^{1}$ & 0 & 0 & 0 & 0 & 0 & 0 & 0 & 0 & 1 & 1 & -1 & -1 \\
$v_{i}^{2}$ & 1 & -1 & -1 & 1 & 0 & 0 & 0 & 0 & 0 & 0 & 0 & 0 \\
$w_{i}^{2}$ & 0 & 0 & 0 & 0 & 1 & 1 & -1 & -1 & 0 & 0 & 0 & 0 \\
$v_{i}^{3}$ & 0 & 0 & 0 & 0 & 0 & 0 & 0 & 0 & 1 & -1 & -1 & 1 \\
$w_{i}^{3}$ & 1 & 1 & -1 & -1 & 0 & 0 & 0 & 0 & 0 & 0 & 0 & 0 \\
\hline
\end{tabular}


12 velocities $\left(\gamma=\gamma_{2}\right)$

\begin{tabular}{|l|rrrrrrrrrrrr|}
\hline & $u_{21}$ & $u_{22}$ & $u_{23}$ & $u_{24}$ & $u_{25}$ & $u_{26}$ & $u_{27}$ & $u_{28}$ & $u_{29}$ & $u_{30}$ & $u_{31}$ & $u_{32}$ \\
\hline$v_{i}^{1}$ & 0 & 0 & 0 & 0 & 0 & 0 & 0 & 0 & 1 & 1 & -1 & -1 \\
$w_{i}^{1}$ & 0 & 0 & 0 & 0 & 1 & -1 & -1 & 1 & 1 & 1 & -1 & -1 \\
$v_{i}^{2}$ & 0 & 0 & 0 & 0 & 1 & 1 & -1 & -1 & 0 & 0 & 0 & 0 \\
$w_{i}^{2}$ & 1 & -1 & -1 & 1 & 1 & 1 & -1 & -1 & 0 & 0 & 0 & 0 \\
$v_{i}^{3}$ & 1 & 1 & -1 & -1 & 0 & 0 & 0 & 0 & 0 & 0 & 0 & 0 \\
$w_{i}^{3}$ & 1 & 1 & -1 & -1 & 0 & 0 & 0 & 0 & 1 & -1 & -1 & 1 \\
\hline
\end{tabular}

12 velocities $\left(\gamma=\gamma_{3}\right)$

\begin{tabular}{|l|rrrrrrrrrrrr|}
\hline & $u_{21}$ & $u_{22}$ & $u_{23}$ & $u_{24}$ & $u_{25}$ & $u_{26}$ & $u_{27}$ & $u_{28}$ & $u_{29}$ & $u_{30}$ & $u_{31}$ & $u_{32}$ \\
\hline$v_{i}^{1}$ & 0 & 0 & 0 & 0 & -1 & 1 & 1 & -1 & 1 & 1 & -1 & -1 \\
$w_{i}^{1}$ & 0 & 0 & 0 & 0 & 1 & -1 & -1 & 1 & 0 & 0 & 0 & 0 \\
$v_{i}^{2}$ & -1 & 1 & 1 & -1 & 1 & 1 & -1 & -1 & 0 & 0 & 0 & 0 \\
$w_{i}^{2}$ & 1 & -1 & -1 & 1 & 0 & 0 & 0 & 0 & 0 & 0 & 0 & 0 \\
$v_{i}^{3}$ & 1 & 1 & -1 & -1 & 0 & 0 & 0 & 0 & -1 & 1 & 1 & -1 \\
$w_{i}^{3}$ & 0 & 0 & 0 & 0 & 0 & 0 & 0 & 0 & 1 & -1 & -1 & 1 \\
\hline
\end{tabular}

12 velocities $\left(\gamma=\gamma_{4}\right)$

\begin{tabular}{|l|rrrrrrrrrrrr|}
\hline & $u_{21}$ & $u_{22}$ & $u_{23}$ & $u_{24}$ & $u_{25}$ & $u_{26}$ & $u_{27}$ & $u_{28}$ & $u_{29}$ & $u_{30}$ & $u_{31}$ & $u_{32}$ \\
\hline$v_{i}^{1}$ & 0 & 0 & 0 & 0 & 1 & -1 & -1 & 1 & 1 & 1 & -1 & -1 \\
$w_{i}^{1}$ & 0 & 0 & 0 & 0 & 1 & -1 & -1 & 1 & 2 & 2 & -2 & -2 \\
$v_{i}^{2}$ & 1 & -1 & -1 & 1 & 1 & 1 & -1 & -1 & 0 & 0 & 0 & 0 \\
$w_{i}^{2}$ & 1 & -1 & -1 & 1 & 2 & 2 & -2 & -2 & 0 & 0 & 0 & 0 \\
$v_{i}^{3}$ & 1 & 1 & -1 & -1 & 0 & 0 & 0 & 0 & 1 & -1 & -1 & 1 \\
$w_{i}^{3}$ & 2 & 2 & -2 & -2 & 0 & 0 & 0 & 0 & 1 & -1 & -1 & 1 \\
\hline
\end{tabular}

Note that

$$
u_{i+2}=-u_{i} \text { for } i=1,2,5,6,9,10,21,22,25,26,29,30
$$

and that

$$
u_{i+4}=-u_{i} \text { for } i=13,14,15,16 \text { 。 }
$$




\section{Appendix 3}

\section{Classification of collisions}

Each equivalence class of collisions is called a type. We have 7 types for $\gamma=\gamma_{1}, \gamma_{2}$, and 6 types for $\gamma=\gamma_{3}, \gamma_{4}$.

$$
\left(\gamma=\gamma_{1}\right)
$$

\begin{tabular}{|c|c|c|c|c|c|c|}
\hline & type & $\begin{array}{c}\text { number of } \\
\text { collisions }\end{array}$ & representative & $E$ & $M$ & $I$ \\
\hline \multirow{3}{*}{$\mathscr{C}^{(1)}$} & $C_{1}$ & 30 & $\left(u_{1}, u_{3}\right) \rightarrow\left(u_{2}, u_{4}\right)$ & $6(1+\tau)$ & 0 & $(-1+2 \tau) / 3$ \\
\hline & $C_{2}$ & 60 & $\left(u_{1}, u_{3}\right) \rightarrow\left(u_{5}, u_{7}\right)$ & $6(1+\tau)$ & 0 & $1 / 3$ \\
\hline & $C_{3}$ & 60 & $\left(u_{1}, u_{6}\right) \rightarrow\left(u_{9}, u_{14}\right)$ & $6(1+\tau)$ & $4(1+\tau)$ & 0 \\
\hline $\mathscr{C}^{(2)}$ & $C_{4}$ & 30 & $\left(u_{21}, u_{23}\right) \rightarrow\left(u_{22}, u_{24}\right)$ & $2(2+\tau)$ & 0 & $(-1+2 \tau) / 5$ \\
\hline \multirow{3}{*}{$\mathscr{C}^{(1,2)}$} & $C_{5}$ & 120 & $\left(u_{1}, u_{23}\right) \rightarrow\left(u_{8}, u_{25}\right)$ & $5+4 \tau$ & 3 & $(15-8 \tau) / 41$ \\
\hline & $C_{6}$ & 120 & $\left(u_{1}, u_{26}\right) \rightarrow\left(u_{2}, u_{25}\right)$ & $5+4 \tau$ & $3+4 \tau$ & $(-27+32 \tau) / 61$ \\
\hline & $C_{7}$ & 120 & $\left(u_{1}, u_{26}\right) \rightarrow\left(u_{5}, u_{22}\right)$ & $5+4 \tau$ & $3+4 \tau$ & $(-5+24 \tau) / 61$ \\
\hline
\end{tabular}

$$
\left(\gamma=\gamma_{2}\right)
$$

\begin{tabular}{|c|c|c|c|c|c|c|}
\hline & type & $\begin{array}{c}\text { number of } \\
\text { collisions }\end{array}$ & representative & $E$ & $M$ & $I$ \\
\hline $\mathscr{C}^{(2)}$ & $C_{4}$ & 30 & $\left(u_{21}, u_{23}\right) \rightarrow\left(u_{22}, u_{24}\right)$ & $2(3+4 \tau)$ & 0 & $(-1+2 \tau) / 5$ \\
\hline & $C_{5}$ & 120 & $\left(u_{1}, u_{23}\right) \rightarrow\left(u_{4}, u_{22}\right)$ & $6+7 \tau$ & $2+\tau$ & $(-5+32 \tau) / 61$ \\
\cline { 2 - 6 } $\mathscr{C}^{(1,2)}$ & $C_{6}$ & 120 & $\left(u_{1}, u_{23}\right) \rightarrow\left(u_{9}, u_{27}\right)$ & $6+7 \tau$ & $2+\tau$ & $(-19+24 \tau) / 61$ \\
\cline { 2 - 6 } & $C_{7}$ & 120 & $\left(u_{1}, u_{25}\right) \rightarrow\left(u_{5}, u_{29}\right)$ & $6+7 \tau$ & $3(2+3 \tau)$ & $(19+10 \tau) / 41$ \\
\hline
\end{tabular}




\begin{tabular}{|c|c|c|c|c|c|c|}
\hline \multicolumn{8}{|c|}{$\left(\gamma=\gamma_{3}\right)$} \\
\hline & type & $\begin{array}{c}\text { number of } \\
\text { collisions }\end{array}$ & representative & $E$ & $M$ & $I$ \\
\hline $\mathscr{C}^{(2)}$ & $C_{4}$ & 30 & $\left(u_{21}, u_{23}\right) \rightarrow\left(u_{22}, u_{24}\right)$ & $2(3-\tau)$ & 0 & $(-1+2 \tau) / 5$ \\
\hline \multirow{\mathscr{C}^{(1,2)}}{*}{} & $C_{5}$ & 60 & $\left(u_{1}, u_{23}\right) \rightarrow\left(u_{16}, u_{26}\right)$ & $2(3+\tau)$ & 4 & $(-1+2 \tau) / 5$ \\
\hline & $C_{6}$ & 60 & $\left(u_{1}, u_{25}\right) \rightarrow\left(u_{13}, u_{22}\right)$ & $2(3+\tau)$ & $4(1+\tau)$ & 0 \\
\hline
\end{tabular}

$$
\left(\gamma=\gamma_{4}\right)
$$

\begin{tabular}{|c|c|c|c|c|c|c|}
\hline & type & $\begin{array}{c}\text { number of } \\
\text { collisions }\end{array}$ & representative & $E$ & $M$ & $I$ \\
\hline $\mathscr{C}^{(2)}$ & $C_{4}$ & 30 & $\left(u_{21}, u_{23}\right) \rightarrow\left(u_{22}, u_{24}\right)$ & $2(7+11 \tau)$ & 0 & $(-1+2 \tau) / 5$ \\
\hline $\mathscr{C}^{(1,2)}$ & $C_{5}$ & 60 & $\left(u_{1}, u_{23}\right) \rightarrow\left(u_{20}, u_{28}\right)$ & $2(5+7 \tau)$ & $4(1+\tau)$ & 0 \\
\hline & $C_{6}$ & 60 & $\left(u_{1}, u_{26}\right) \rightarrow\left(u_{20}, u_{21}\right)$ & $2(5+7 \tau)$ & $4(2+3 \tau)$ & $(-1+2 \tau) / 5$ \\
\hline
\end{tabular}

\section{Appendix 4}

\section{Collision term}

We give here the concrete forms of $Q_{1}(F, F)$ and $Q_{21}(F, F)$ for $\gamma=\gamma_{1}, \gamma_{3}$. The other $Q_{i}$ 's are obtained by suitable permutation of indices. The case of $\gamma=\gamma_{2}, \gamma_{4}$ is omitted.

Case: $\gamma=\gamma_{1}$.

$$
\begin{aligned}
Q_{1}(F, F)= & a_{1}\left\{\left(F_{2} F_{4}-F_{1} F_{3}\right)+\left(F_{13} F_{17}-F_{1} F_{3}\right)+\left(F_{16} F_{20}-F_{1} F_{3}\right)\right\} \\
+ & a_{2}\left\{\left(F_{5} F_{7}-F_{1} F_{3}\right)+\left(F_{6} F_{8}-F_{1} F_{3}\right)+\left(F_{9} F_{11}-F_{1} F_{3}\right)\right. \\
& \left.+\left(F_{10} F_{12}-F_{1} F_{3}\right)+\left(F_{14} F_{18}-F_{1} F_{3}\right)+\left(F_{15} F_{19}-F_{1} F_{3}\right)\right\} \\
+ & a_{3}\left\{\left(F_{9} F_{14}-F_{1} F_{6}\right)+\left(F_{10} F_{15}-F_{1} F_{7}\right)+\left(F_{8} F_{14}-F_{1} F_{11}\right)\right. \\
& \left.+\left(F_{5} F_{15}-F_{1} F_{12}\right)+\left(F_{8} F_{9}-F_{1} F_{18}\right)+\left(F_{5} F_{10}-F_{1} F_{19}\right)\right\} \\
+ & a_{5}\left\{\left(F_{8} F_{25}-F_{1} F_{23}\right)+\left(F_{10} F_{32}-F_{1} F_{23}\right)+\left(F_{5} F_{28}-F_{1} F_{24}\right)\right. \\
& \left.+\left(F_{9} F_{32}-F_{1} F_{24}\right)+\left(F_{14} F_{28}-F_{1} F_{31}\right)+\left(F_{15} F_{25}-F_{1} F_{31}\right)\right\} \\
+ & a_{6}\left\{\left(F_{2} F_{25}-F_{1} F_{26}\right)+\left(F_{13} F_{32}-F_{1} F_{26}\right)+\left(F_{2} F_{28}-F_{1} F_{27}\right)\right. \\
& \left.+\left(F_{16} F_{32}-F_{1} F_{27}\right)+\left(F_{13} F_{28}-F_{1} F_{30}\right)+\left(F_{16} F_{25}-F_{1} F_{30}\right)\right\} \\
+ & a_{7}\left\{\left(F_{5} F_{22}-F_{1} F_{26}\right)+\left(F_{14} F_{29}-F_{1} F_{26}\right)+\left(F_{8} F_{21}-F_{1} F_{27}\right)\right. \\
& \left.+\left(F_{15} F_{29}-F_{1} F_{27}\right)+\left(F_{9} F_{22}-F_{1} F_{30}\right)+\left(F_{10} F_{21}-F_{1} F_{30}\right)\right\},
\end{aligned}
$$




$$
\begin{aligned}
Q_{21}(F, F)=a_{4} & \left\{\left(F_{22} F_{24}-F_{21} F_{23}\right)+\left(F_{25} F_{27}-F_{21} F_{23}\right)+\left(F_{26} F_{28}-F_{21} F_{23}\right)\right. \\
& \left.+\left(F_{29} F_{31}-F_{21} F_{23}\right)+\left(F_{30} F_{32}-F_{21} F_{23}\right)\right\} \\
+a_{5} & \left\{\left(F_{6} F_{27}-F_{3} F_{21}\right)+\left(F_{12} F_{30}-F_{3} F_{21}\right)+\left(F_{6} F_{28}-F_{4} F_{21}\right)\right. \\
& +\left(F_{9} F_{31}-F_{4} F_{21}\right)+\left(F_{15} F_{30}-F_{7} F_{21}\right)+\left(F_{16} F_{31}-F_{7} F_{21}\right) \\
& +\left(F_{12} F_{28}-F_{17} F_{21}\right)+\left(F_{15} F_{24}-F_{17} F_{21}\right)+\left(F_{9} F_{27}-F_{18} F_{21}\right) \\
& \left.+\left(F_{16} F_{24}-F_{18} F_{21}\right)\right\} \\
+a_{6} & \left\{\left(F_{15} F_{29}-F_{8} F_{21}\right)+\left(F_{16} F_{32}-F_{8} F_{21}\right)+\left(F_{9} F_{22}-F_{10} F_{21}\right)\right. \\
& +\left(F_{16} F_{25}-F_{10} F_{21}\right)+\left(F_{12} F_{22}-F_{11} F_{21}\right)+\left(F_{15} F_{26}-F_{11} F_{21}\right) \\
& +\left(F_{6} F_{29}-F_{19} F_{21}\right)+\left(F_{9} F_{26}-F_{19} F_{21}\right)+\left(F_{6} F_{32}-F_{20} F_{21}\right) \\
& \left.+\left(F_{12} F_{25}-F_{20} F_{21}\right)\right\} \\
+a_{7} & \left\{\left(F_{1} F_{27}-F_{8} F_{21}\right)+\left(F_{2} F_{28}-F_{8} F_{21}\right)+\left(F_{1} F_{30}-F_{10} F_{21}\right)\right. \\
& +\left(F_{13} F_{28}-F_{10} F_{21}\right)+\left(F_{2} F_{31}-F_{11} F_{21}\right)+\left(F_{14} F_{27}-F_{11} F_{21}\right) \\
& +\left(F_{5} F_{30}-F_{19} F_{21}\right)+\left(F_{13} F_{24}-F_{19} F_{21}\right)+\left(F_{5} F_{31}-F_{20} F_{21}\right) \\
& \left.+\left(F_{14} F_{24}-F_{20} F_{21}\right)\right\} .
\end{aligned}
$$

Case: $\gamma=\gamma_{3}$.

$$
\begin{aligned}
Q_{1}(F, F)= & a_{1}\left\{\left(F_{2} F_{4}-F_{1} F_{3}\right)+\left(F_{13} F_{17}-F_{1} F_{3}\right)+\left(F_{16} F_{20}-F_{1} F_{3}\right)\right\} \\
+ & a_{2}\left\{\left(F_{5} F_{7}-F_{1} F_{3}\right)+\left(F_{6} F_{8}-F_{1} F_{3}\right)+\left(F_{9} F_{11}-F_{1} F_{3}\right)\right. \\
& \left.+\left(F_{10} F_{12}-F_{1} F_{3}\right)+\left(F_{14} F_{18}-F_{1} F_{3}\right)+\left(F_{15} F_{19}-F_{1} F_{3}\right)\right\} \\
+ & \left\{\left(F_{9} F_{14}-F_{1} F_{6}\right)+\left(F_{10} F_{15}-F_{1} F_{7}\right)+\left(F_{8} F_{14}-F_{1} F_{11}\right)\right. \\
& \left.+\left(F_{5} F_{15}-F_{1} F_{12}\right)+\left(F_{8} F_{9}-F_{1} F_{18}\right)+\left(F_{5} F_{10}-F_{1} F_{19}\right)\right\} \\
+ & a_{5}\left\{\left(F_{13} F_{27}-F_{1} F_{23}\right)+\left(F_{16} F_{26}-F_{1} F_{24}\right)+\left(F_{2} F_{30}-F_{1} F_{31}\right)\right\} \\
+ & a_{6}\left\{\left(F_{13} F_{22}-F_{1} F_{25}\right)+\left(F_{16} F_{21}-F_{1} F_{28}\right)+\left(F_{2} F_{29}-F_{1} F_{32}\right)\right\}, \\
Q_{21}(F, F)=a_{4}\left\{\left(F_{22} F_{24}-F_{21} F_{23}\right)+\left(F_{25} F_{27}-F_{21} F_{23}\right)+\left(F_{26} F_{28}-F_{21} F_{23}\right)\right. & \left.+\left(F_{29} F_{31}-F_{21} F_{23}\right)+\left(F_{30} F_{32}-F_{21} F_{23}\right)\right\} \\
+a_{5}\{( & \left(F_{20} F_{28}-F_{3} F_{21}\right)+\left(F_{19} F_{27}-F_{4} F_{21}\right)+\left(F_{8} F_{24}-F_{7} F_{21}\right) \\
& \left.+\left(F_{11} F_{30}-F_{17} F_{21}\right)+\left(F_{10} F_{31}-F_{18} F_{21}\right)\right\} \\
+ & a_{6}\left\{\left(F_{5} F_{24}-F_{6} F_{21}\right)+\left(F_{13} F_{20}-F_{9} F_{21}\right)+\left(F_{14} F_{31}-F_{12} F_{21}\right)\right. \\
& \left.+\left(F_{2} F_{27}-F_{15} F_{21}\right)+\left(F_{1} F_{28}-F_{16} F_{21}\right)\right\} .
\end{aligned}
$$

\section{Appendix 5}

\section{Classification of $\boldsymbol{P}$-sets}

In what follows, we say simply classes in place of equivalence classes. The set of integers in bracket denotes a respresentative. 
Case: $\gamma=\gamma_{1}$.

$\left.1^{\circ}\right) \mathscr{Q}^{(3)}$ consists of 19 classes.

(a) 3 classes containing 20 P-sets: $\{1,5,9\},\{21,22,29\},\{21$, $24,27\}$.

(b) 11 classes containing $60 P$-sets: $\{1,2,6\},\{1,4,11\},\{1,5,11\}$, $\{1,2,21\},\{1,2,23\},\{1,4,26\},\{1,5,23\},\{1,5,30\},\{1,21,22\}$, $\{1,23,24\},\{1,26,27\}$.

(c) 5 classes containing $120 P$-sets: $\{1,6,22\},\{1,6,23\},\{1,6,26\}$, $\{1,6,28\},\{1,6,30\}$.

$\left.2^{\circ}\right) \mathscr{Q}^{(4)}$ consists of 7 classes.

(a) 2 classes containing $30 P$-sets: $\{1,3,5,7\},\{1,4,25,28\}$.

(b) 5 classes containing $60 P$-sets: $\{1,2,25,26\},\{1,4,21,24\}$, $\{1,5,22,26\},\{1,5,24,28\},\{1,5,27,31\}$.

$\left.3^{\circ}\right) \mathscr{Q}^{(5)}$ consists of 3 classes, each of which contains $12 P$-sets: $\{1,2,5,13,14\},\{1,4,9,15,17\},\{21,22,25,28,30\}$.

$\left.4^{\circ}\right) \mathscr{Q}^{(6)}$ consists of 2 classes.

(a) one classe containing $20 P$-sets: $\{1,2,9,12,19,20\}$.

(b) one classe containing $30 P$-sets: $\{1,5,10,19,25,29\}$.

$\left.5^{\circ}\right) \mathscr{2}^{(8)}$ consists of only one class containing $15 P$-sets:

$\{1,2,3,4,29,30,31,32\}$.

Case: $\gamma=\gamma_{2}$.

$\left.1^{\circ}\right) \mathscr{Q}^{(3)}$ consists of 19 classes.

(a) 3 classes containing $20 P$-sets: $\{1,5,9\},\{21,22,29\},\{21$, $24,27\}$.

(b) 11 classes containing $60 P$-sets: $\{1,2,6\},\{1,2,23\},\{1,4$, $11\},\{1,4,25\},\{1,4,26\},\{1,5,11\},\{1,5,23\},\{1,5,32\}$, $\{1,23,24\},\{1,25,28\},\{1,26,27\}$.

(c) 5 classes containg $120 P$-sets: $\{1,6,21\},\{1,6,22\},\{1,6$, $23\},\{1,6,28\},\{1,6,30\}$.

$\left.2^{\circ}\right) \mathscr{Q}^{(4)}$ consists of 7 classes.

(a) 2 classes containing $30 P$-sets: $\{1,2,21,22\},\{1,3,5,7\}$.

(b) 5 classes containing $60 P$-sets: $\{1,2,25,26\},\{1,4,21,24\}$, $\{1,5,24,28\},\{1,5,25,29\},\{1,5,27,31\}$ 。

$\left.3^{\circ}\right) \mathscr{2}^{(5)}$ consists of 3 classes, each of which contains $12 P$-sets: $\{1,2,5,13,14\},\{1,4,9,15,17\},\{21,22,25,28,30\}$.

$\left.4^{\circ}\right) \mathscr{Q}^{(6)}$ consists of 2 classes. 
(a) one class containing $20 P$-sets: $\{1,2,9,12,19,20\}$.

(b) one class containing $30 P$-sets: $\{1,5,10,19,21,30\}$.

$\left.5^{\circ}\right) \mathscr{Q}^{(8)}$ consists of only one class containg $15 P_{\text {-sets }}:\{1,2,3,4$, $29,30,31,32\}$ 。

Case: $\gamma=\gamma_{3}$

$\left.1^{\circ}\right) \mathscr{Q}^{(3)}$ consisits of 9 classes.

(a) 2 classes containing $20 P$-sets: $\{1,5,9\},\{21,24,27\}$.

(b) 6 classes containing $60 P$-sets: $\{1,2,6\},\{1,2,23\}$, $\{1,5,11\},\{1,5,23\},\{1,5,32\},\{1,25,28\}$.

(c) one class containing $120 P$-sets: $\{1,6,28\}$.

$\left.2^{\circ}\right) \mathscr{Q}^{(4)}$ consists of 3 classes.

(a) 2 classes containing $30 P$-sets: $\{1,3,5,7\},\{1,5,10,19\}$.

(b) one class containing $60 P$-sets: $\{1,5,24,28\}$.

$\left.3^{\circ}\right) \mathscr{Q}^{(5)}$ consists of 2 classes.

(a) one class containing $12 P$-sets: $\{1,2,5,13,14\}$.

(b) one class containing $60 P$-sets: $\{1,4,11,29,30\}$.

$\left.4^{\circ}\right) \mathscr{Q}^{(8)}$ consists of only one class containing $15 P$-sets: $\{1,2,3,4$, $29,30,31,32\}$.

$\left.5^{\circ}\right) \mathscr{Q}^{(9)}$ consists of only one class containing $20 P$-sets: $\{1,2,9$, $12,19,20,21,25,26\}$.

$\left.6^{\circ}\right) \mathscr{Q}^{(10)}$ consists of only one class containing $12 P$-sets: $\{1,4,9$, $15,17,22,23,27,29,30\}$.

Case: $\gamma=\gamma_{4}$

$\left.1^{\circ}\right) \mathscr{Q}^{(3)}$ consists of 9 classes.

(a) 2 classes containing $20 P$-sets: $\{1,5,9\},\{21,22,29\}$ 。

(b) 6 classes containing $60 P$-sets: $\{1,4,11\},\{1,4,26\},\{1,5$, $11\},\{1,5,23\},\{1,5,30\},\{1,23,24\}$.

(c) one class containing $120 P$-sets: $\{1,6,23\}$.

$\left.2^{\circ}\right) \mathscr{Q}^{(4)}$ consists of 3 classes.

(a) 2 classes containing $30 P$-sets: $\{1,3,5,7\},\{1,5,10,19\}$.

(b) one class containing $60 P$-sets: $\{1,5,27,31\}$.

$\left.3^{\circ}\right) \mathscr{Q}^{(5)}$ consists of 2 classes.

(a) one class containing $12 P$-sets: $\{1,4,9,15,17\}$.

(b) one class containing $60 P$-sets: $\{1,2,6,29,32\}$.

$\left.4^{\circ}\right) \mathscr{Q}^{(8)}$ consists of only one class containing $15 P$-sets: $\{1,2,3,4$, $29,30,31,32\}$. 
$\left.5^{\circ}\right) \mathscr{Q}^{(9)}$ consists of only one class containing $20 P$-sets: $\{1,2,9$, $12,19,20,24,29,32\}$.

$\left.6^{\circ}\right) \mathscr{Q}^{(10)}$ consists of only one class containing $12 \quad P$-sets: $\{1,2,5$, $13,14,22,25,26,29,32\}$.

\section{References}

[1] Cabannes, H., Solution global du problème de Cauchy en théorie cinétique discrète, J. Mécanique, 17 (1978), 1-22.

[2] Solution global de l'équation de Boltzmann discrète pour les modèles spatiaux réguliers à 12 ou 20 vitesses, Mech. Res. Comm., 10 (1983), 317-322.

[3] - private communication.

[4] The regular space models in discrete kinetic theory (to appear in Mech. Res. Comm.).

[5] Cercignani, C., Sur des critères d'existence global en théorie cinétique discrète $C . R$. Acad. Sci. Paris Sér. I, 301 (1985), 89-92.

[6] Coxeter, H. S. M., Introduction to Geometry, 2nd epition, John Wiley \& Sons, New York, 1965.

[7] Gatignol, R., Théorie Cinétique des Gas à Répartition Discrète de Vitesses, Lecture Note in Physics 36, Springer, Berlin-Heidelberg-New York, 1975.

[8] Kawashima, S., Global existence and stability of solutions for discrete velocity models of the Boltzmann equation, Lecture Note in Numerical and Applied Analysis 6, 59-85, Kinokuniya, Tokyo, 1984.

[9] Shizuta, Y. and Kawashima, S., Systems of equations of hyperbolic-parabolic type with applications to the discrete Boltzmann equation, Hokkaido Math. J. 14 (1985), 249-275.

[10] The regularity of discrete models of the Boltzmann equation, Proc. Japan Acad., Ser. A, Math. Sci., 61 (1985), 252-254.

[11] The regular discrete models of the Boltzmann equation, Recent Developments in Mathematical Analysis and Partial Differential Equations (Papers Dedicated to Professor Sigeru Mizohata on the Occasion of His Sixtieth Birthday), 1985. 
